\title{
Steroid response pattern and outcome of pediatric idiopathic nephrotic syndrome: a single-center experience in northwest Iran
}

This article was published in the following Dove Press journal:

Therapeutics and Clinical Risk Management

25 May $201 \mathrm{I}$

Number of times this article has been viewed

\author{
Fakhrossadat Mortazavi ${ }^{1,2}$ \\ Yaser Soleimani Khiavi ${ }^{2}$ \\ 'Department of Pediatric Nephrology, \\ Tabriz University of Medical Sciences, \\ Tabriz, Iran; ${ }^{2}$ Pediatric Health \\ Research Center, Tabriz University \\ of Medical Sciences, Tabriz, Iran
}

Aims: Characteristics of nephrotic syndrome (NS) in children varies in different geographical areas based on genetic and environmental factors. The aim of this study is to evaluate the steroid response pattern and outcome of idiopathic NS (INS) in a pediatric referral hospital in northwest Iran.

Methods: Medical records of all admitted children under 14 years of age with INS in the Children's Hospital of Tabriz, Iran, from 1999 to 2010 were studied retrospectively. Demographics, pattern of response to medications, recurrence rate, histopathology, and outcome were documented.

Results: A total of 165 patients with INS, with a mean age of $4.98 \pm 2.61$ years were studied. Male to female ratio was 2:1. Duration of follow-up was $5.36 \pm 2.2$ years ( $1-10$ years). A total of 124 patients $(75.2 \%)$ responded to steroids, and 41 patients $(24.8 \%)$ were steroid resistant. Frequency of hematuria $(P=0.01)$ and steroid resistance $(P=0.005)$ in girls was significantly higher than boys. Patients with steroid resistance had a higher frequency of hematuria $(P=0.001)$ and a higher mean age $(P=0.017)$ in comparison with steroid responders. Renal biopsy carried out in 49 patients $(29.7 \%)$ revealed minimal change in NS in $20(40.8 \%)$, focal segmental glomerulosclerosis in $16(32.7 \%)$, and mesangial proliferation in $11(22.5 \%)$ patients. Twenty-two steroid resistant patients $(13 \%)$ achieved remission with other immunosupressives. Nineteen patients $(11.5 \%)$ were resistant to all treatment modalities; of these, nine $(5.4 \%)$ progressed to end-stage renal disease, and $10(6 \%)$ continued nephrotic range proteinuria. Seven patients (4.2\%) died. Of the 146 patients who achieved remission with any one of the treatment modalities, 91 patients $(62.3 \%)$ experienced at least one recurrence episode, 15 patients $(10.3 \%)$ were frequent relapsers, and 12 patients $(8.2 \%)$ were steroid dependent. Higher age at onset of NS was associated with lower relapse rate $(P=0.04)$.

Conclusion: Demographics, histological features, and outcome of INS in our area were similar to western countries. In the present study, risk of steroid resistance was higher in girls than boys.

Keywords: nephrotic syndrome, steroid resistance, relapse

\section{Introduction}

The incidence of nephrotic syndrome (NS) is estimated to be 2-7 cases per 100000 children per year, ${ }^{1-4}$ and its cumulative prevalence rate is 16 per 100000 children below age of $16 .^{2} \mathrm{NS}$ is 15 times more common in children than adults. ${ }^{1}$ Approximately $90 \%$ of children with NS have idiopathic NS (INS), and the remaining $10 \%$ have secondary NS, related to infections, systemic diseases, malignancy, and other glomerular diseases. Minimal change nephrotic syndrome (MCNS) accounts for $85 \%$ of INS, and more than $95 \%$ of these respond to steroid therapy and don't need renal biopsy. ${ }^{1,2}$ Children
Correspondence: Fakhrossadat Mortazavi Children's Hospital of Tabriz, Sheshgelan Street, Tabriz, Iran

Tel +98 4l I 5262280;

$+989141166729$

Fax +98 4II 5262279

Email mortazavi_fakhri@yahoo.co.uk 
with steroid-sensitive NS (SSNS), have a benign prognosis with good preservation of long-term kidney function. Steroid resistance is associated with a high risk of developing chronic kidney disease. Focal segmental glomerulosclerosis (FSGS) is the main cause of steroid resistant NS (SRNS) ${ }^{5}$ and accounts for $10 \%-20 \%$ of end-stage renal disease (ESRD) in children. ${ }^{6}$ Previous studies emphasize the considerable influence of racial and geographical factors on steroid response and histological pattern and outcome of INS. ${ }^{7}$ Moreover, there are some reports indicating the changing face of childhood INS with time. ${ }^{8,9}$ Recent studies show that the frequency of FSGS in children has dramatically increased over the past two decades in some parts of world. ${ }^{9-11}$ There are few data about the characteristics of INS in our area. The aim of this study is to evaluate the steroid response pattern and longterm outcome of pediatric INS in a tertiary care hospital in northwest Iran and compare it with other studies.

\section{Materials and methods}

Medical records of all admitted children under 14 years of age with NS in the Children's Hospital of Tabriz, Iran, from 1999 to 2010 were studied retrospectively. The Children's Hospital of Tabriz is the largest pediatric referral center in northwest Iran, and most nephrotic children are referred to this hospital. NS was defined as a constellation of proteinuria more than $40 \mathrm{mg} / \mathrm{m}^{2} /$ day, hypoalbuminemia (serum albumin $<2.5 \mathrm{~g} / \mathrm{dL}$ ), hyperlipidemia (serum cholesterol $>250 \mathrm{mg} / \mathrm{dL}$ ), and edema. INS was defined as NS in children between 6 months and 14 years without a systemic illness causing NS, with normal creatinine, $\mathrm{C}_{3}$, and $\mathrm{C}_{4}$ levels, which either responded to steroids or had a histological diagnosis of INS (MCNS or FSGS or mesangial proliferation in renal biopsy). Patients with IgA nephropathy, membranoproliferative glomerulonephritis, membranous nephropathy, congenital NS, chronic renal failure, and patients with inadequate data were excluded. Serum urea, creatinine, cholesterol, triglyceride, total protein, albumin, serum electrolytes, HBsAg, serum complement levels of $\mathrm{C}_{3}, \mathrm{C}_{4}$, ANA, urine analysis, urine culture, 24-hour urine protein excretion, and sonography of kidneys were carried out in all patients at the time of first admission. Indications that were considered for renal biopsy included: resistance to steroids, gross hematuria, age less than 1 year and more than 11 years, low serum level of $\mathrm{C} 3$ and $\mathrm{C} 4$, and persistent hypertension. Frequent relapsers and steroid-dependent patients underwent renal biopsy before initiation of cyclosporine. Informed consent was obtained from parents before renal biopsy. The biopsy specimens were examined by light and immunofluorescence microscopy.
Patients who had no indication for renal biopsy were treated with prednisolone $60 \mathrm{mg} / \mathrm{m}^{2} /$ day for 4-6 weeks followed by prednisolone $40 \mathrm{mg} / \mathrm{m}^{2}$ on alternate days for a further 4 weeks. The prednisolone dose was then tapered and discontinued over the next 2-3 months. Steroid resistants, frequent relapsers, and steroid dependants underwent treatment with other alternative agents including levamisol (2-3 mg/kg/day), cyclophosphamide (2-3 mg/kg/day for 8-12 months), cyclosporine (3-6 mg/kg/day), mycophenolate mofetil (600 mg/m² dose bid) and pulse methylprednisolone (30 mg/ $\mathrm{kg}$ bolus with the first six doses given every other day followed by a tapering regimen for periods of up to 18 months). All patients were followed up at the out-patient service at intervals of 1 week to 1 month by the same nephrologists. Data including: sex, age of NS onset, clinical and laboratory findings, results of renal biopsy, pattern of response to steroids and other alternative agents, duration of follow-up, and outcome were collected in designed forms.

The following definitions were considered for classification of response pattern to steroid therapy. ${ }^{1,2}$

1. Steroid response: complete resolution of proteinuria within 4-6 weeks of steroid therapy.

2. Relapse: occurrence of $3^{+}-4^{+}$proteinuria plus edema.

3. Frequent relapse: three or more relapses within 12 months.

4. Steroid dependence: occurrence of relapse during tapering of steroid dose or within 28 days of stopping steroid therapy.

5. Steroid resistance: failure to respond to prednisolone therapy within 6-8 weeks.

This study was approved by the ethical committee of Tabriz University of Medical Sciences. Statistical package for the social sciences (SPSS) version 16 was used for data analysis. Quantitative variables were presented as mean \pm standard deviation. For comparison of differences between qualitative variables, chi-square or exact Fisher's tests were used, and for comparison of quantitative variables, nonparametric Mann-Whitney $U$ test was used. A $P$ value less than 0.05 was considered significant.

\section{Results}

From October 1999 to October 2010, 226 patients with NS admitted to the Children's Hospital of Tabriz. Fifty-two $(23 \%)$ patients with secondary NS and nine patients with incomplete data were excluded from the study, and 165 $(73 \%)$ patients with diagnosis of INS were studied. The mean duration of follow-up period was $5.36 \pm 2.2$ years (range 1-10 years). Mean age of patients at initial presentation 
was $4.98 \pm 2.61$ years (range 10 months -12 years), with a peak incidence at 3-6 years of age (Figure 1). None of the patients were positive for HBsAg.

Hematuria was detected in $40(24.2 \%)$ patients. Thirty-three patients had microscopic hematuria (20\%) and 7 (4.2\%) patients had gross hematuria. Hypertension was found in 19 (11.5\%) patients. There were 110 boys and 55 girls (male/ female $=2$ ). Table 1 shows the characteristics of boys in comparison with girls. Frequency of hematuria $(P=0.01)$ and steroid resistance $(P=0.005)$ in girls was significantly higher than boys.

A total of 124 patients $(75.2 \%)$ responded to standard therapy with steroids, and 41 patients $(24.8 \%)$ were steroid resistant. Patients with SRNS had a higher frequency of hematuria $(P=0.001)$ and a higher mean age $(P=0.017)$ compared with the SSNS group (Table 2). After dividing the patients into four age groups, we found that age of NS onset has a positive predictive ability in occurrence of steroid resistance (odds ratio $[\mathrm{OR}]=1.476$, confidence interval $[\mathrm{CI}]=1.016-2.144)$. So probability of steroid resistance increases with age of NS onset $(P=0.04)$.

Renal biopsy was carried out in 49 (29.7\%) patients, including 40 patients with SRNS and nine frequent relapser patients. The histopathologic results were: MCNS in 20 (40.8\%), FSGS in $16(32.7 \%)$, diffuse mesangial proliferation (DMP) in $11(22.5 \%)$, and focal mesangial proliferation in two (4\%) patients. Six patients with DMP which revealed slight IgM deposition in immunofluorescence microscopy diagnosed as IgM nephropathy.

A total of 10 out of 41 (24.4\%) patients with SRNS responded to cyclophosphamide, seven (17.1\%) patients responded to pulse therapy with methylprednisolone, and five $(12.2 \%)$ patients achieved remission with cyclosporine. A total of 19 patients were resistant to all treatment modalities ( $46.3 \%$ of steroid resistants and $11.5 \%$ of total study population); of these, nine (5.4\%) progressed to ESRD (six patients

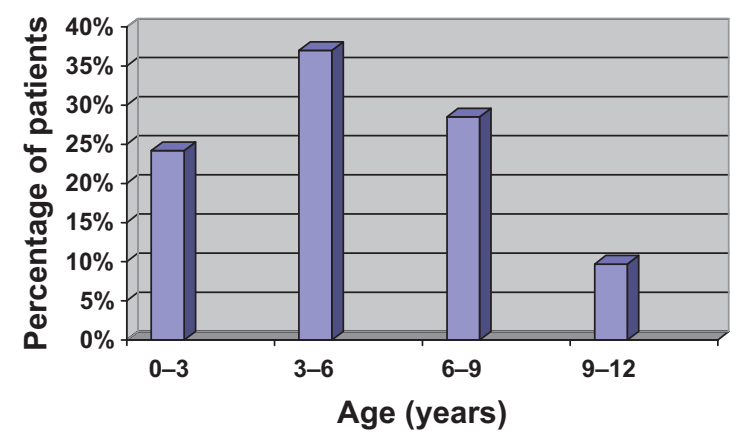

Figure I Age distribution of 165 patients with idiopathic nephrotic syndrome.
Table I Comparison of characteristics of INS between boys and girls

\begin{tabular}{lllll}
\hline Variable & Boys & Girls & $P$ value & Total \\
\hline Number (\%) & II $(66.7 \%)$ & $55(33.3 \%)$ & - & $165(100 \%)$ \\
Hematuria & $19(17.3 \%)$ & $21(38.2 \%)$ & $P=0.01$ & $40(24.2 \%)$ \\
Hypertension & $12(10.9 \%)$ & $7(12.7 \%)$ & $P=0.46$ & $19(11.6 \%)$ \\
Steroid resistance & $18(16.4 \%)$ & $23(42 \%)$ & $P=0.005$ & $41(24.8 \%)$ \\
Progression to & $4(3.6 \%)$ & $5(9 \%)$ & $P=0.14$ & $9(5.4 \%)$ \\
ESRD & & & & \\
Mortality & $\mathrm{I}(0.9 \%)$ & $6(10.9 \%)$ & $P=0.09$ & $7(4.4 \%)$ \\
\hline
\end{tabular}

Abbreviations: ESRD, end-stage renal disease; INS, idiopathic nephrotic syndrome.

with FSGS and three with IgM nephropathy), and 10 patients (6\%) continued nephrotic range proteinuria.

A total of 91 out of 146 patients $(62.3 \%)$ who achieved remission with any one of the treatment modalities experienced at least one recurrence during the follow-up period. Fifteen patients $(10.3 \%)$ were frequent relapsers, and 12 patients $(8.2 \%)$ were steroid dependent. There was not any significant difference in frequency of hematuria $(P=0.16)$, hypertension $(P=0.24)$, and male sex $(P=0.24)$ between relapser and nonrelapser patients. Probability of recurrence decreased with increasing age of NS onset $(\mathrm{OR}=0.696$, $\mathrm{CI}=0.487-0.995, P=0.04)$.

Mortality occurred in $7(4.38 \%)$ patients (Table 1$)$. Five patients died due to complications of NS (including infections in four patients and cerebral thrombotic event in one patient), and two patients with ESRD died due to heart failure.

\section{Discussion}

In the present study we reviewed the demographics, steroid response pattern, and outcome of INS in children. We found a male/female ratio of $2: 1$, which is similar to earlier studies which show a male preponderance. ${ }^{1,2}$ In Western countries, SSNS is the most common type seen in children. ${ }^{1,2}$ Response to initial steroid therapy has been reported from $77.6 \%$ to $88.0 \%$ in various studies..$^{4,7,12-14}$ In our study,

Table 2 Comparison of children with steroid-responsive and steroid-resistant idiopathic nephrotic syndrome

\begin{tabular}{|c|c|c|c|}
\hline Variable & Steroid sensitive & Steroid resistant & $P$ value \\
\hline Number (\%) & $124(75.2 \%)$ & $4 \mathrm{I}(24.8 \%)$ & - \\
\hline Boys & 91 (73.4\%) & $19(46.3 \%)$ & - \\
\hline Girls & $33(26.6 \%)$ & $22(53.7 \%)$ & $P=0.005$ \\
\hline Hematuria & $19(17.3 \%)$ & $21(51.2 \%)$ & $P=0.001$ \\
\hline Hypertension & $13(10.5 \%)$ & $6(14.6 \%)$ & $P=0.3$ \\
\hline Mean age (years) & $4.6 \pm 2.4$ & $5.8 \pm 3.2$ & $P=0.017$ \\
\hline $\begin{array}{l}\text { Mean 24-hour } \\
\text { urine protein }(\mathrm{mg})\end{array}$ & $3190.3 \pm 322.34$ & $4157.78 \pm 448.86$ & $P=0.11$ \\
\hline
\end{tabular}


$75.2 \%$ of patients responded to standard steroid therapy. While in black children of South Africa, steroid response even in MCNS was less then 50\%. ${ }^{15}$ Also, in Ghana steroid responsiveness was reported in $50 \%$ of children with INS. ${ }^{16}$ A study by Gulati et al showed that male sex, age more than 8 years at onset of NS, and presence of hematuria were predictors of resistance to steroids. ${ }^{14}$ Also, in our study, mean age and frequency of hematuria were significantly higher in the SRNS than the SSNS group. However, the frequency of girls was significantly higher than boys in the SRNS group compared with the SSNS group (Table 2). This finding is in contradiction with most previous studies, which show a male preponderance both in SRNS and SSNS patients. ${ }^{10,17,18}$

SRNS is a problematic disease with a poor outcome and a high rate of progression to ESRD. In the present study $22 \%$ (9/41) of patients with SRNS reached ESRD, which is similar to the study of Otukesh et al in Tehran, Iran, who reported a $26 \%$ progression to ESRD in children with SRNS. ${ }^{18}$ In a study by Mekahli et al, the renal survival rate after 5, 10, and 15 years was $75 \%, 58 \%$, and $53 \%$ respectively. ${ }^{17}$ The lower incidence in our study may be attributed to a shorter follow-up period than in the Mekahli study.

Risk factors of progression to ESRD in INS are not well known. A study by Chang et al showed that age of beyond 8 years at onset of NS is associated with a higher incidence of hypertension, hematuria, and progression to ESRD. ${ }^{19}$ Mekahli et al reported that an age of more than 10 years at NS onset was the only independent predictor of ESRD. ${ }^{17}$ Some studies focus on histopathology, and FSGS is generally believed to carry the worst prognosis. ${ }^{20}$ FSGS accounts for only $10 \%$ of INS in children, ${ }^{1,2}$ but its frequency in different studies ranges from $7 \%$ to $42 \%$ based on different criteria considered for renal biopsy. ${ }^{7,13,17,19,21}$ In our study, FSGS accounts for $32.7 \%$ of renal biopsies, which is within the reported range by other studies. Frequency of ESRD in patients with FSGS ranges from $13 \%-78 \%$ in various studies, depending on the follow-up period. ${ }^{20}$ In the present study, 6 out of 20 patients with FSGS (33\%) reached ESRD, which is in accordance with the literature. However, many studies indicate that steroid responsiveness is more accurate in predicting outcome than histopathological diagnosis. ${ }^{4,22,23}$ Otukesh et al found that achievement of complete or partial remission with any immunosuppressive drug reduces the risk of ESRD. ${ }^{18}$ In our study, not one of the patients who achieved remission with any one of the treatment modalities progressed to ESRD, and all patients who reached ESRD were resistant to all kinds of treatment modalities.
Childhood INS is characterized by a relapsing course. Rate of relapse has been reported from $60.5 \%$ to $84.0 \%$ in previous studies. $3,4,7,12,22$ We found a relapse rate of $62 \%$ in our patients, which is in accordance with the literature. The frequency of relapse has been noted to be higher at a younger age (less than 3-4 years) at onset of NS. ${ }^{16}$ The present study also showed that the rate of recurrence decreased with increasing age of NS onset $(P=0.04)$. A study by Noer showed that the time interval between early steroid response and the first relapse, number of relapses within the first 6 months, hematuria, and male sex were the significant predictors of relapse. ${ }^{24}$ However, we didn't find any significant difference in frequency of hematuria and male sex between relapsers and nonrelapsers.

Mortality of NS has dramatically diminished in recent years with the introduction of effective therapies. The overall mortality has been reported at $2.2 \%-3.1 \% .{ }^{12,15}$ However, mortality of our patients seems to be higher than the literature, which may be explained by poor compliance of our patients or limited facilities to prevent infections.

\section{Conclusion}

Sex and age distribution, histological features, steroid response pattern, and outcome of INS in our study were in accordance with Western countries and Turkey. In this study, higher age at onset of NS and hematuria were found to be a risk factor for steroid resistance, and lower age at onset of NS increased the risk of recurrence. Also, our study showed a higher frequency of steroid resistance in girls than boys. Although the frequency of progression to ESRD and mortality in girls was higher than boys, it was not statistically significant. These findings should be evaluated by other multicenter investigations.

\section{Disclosure}

The authors report no conflicts of interest in this work.

\section{References}

1. Vogt BA, Avner ED. Conditions particularly associated with proteinuria. In: Kliegman RM, Behrman RE, Jenson HB, Stanton BF. Nelson Text Book of Pediatrics. 18th ed. Philadelphia, PA: Saunders; 2007:2190-2195.

2. Niaudet P, Boyer O. Idiopathic nephrotic syndrome in childhood: clinical aspects. In: Avner ED, Harmon WE, Niaudet P, Yoshikawa N. Pediatric Nephrology. 6th ed. Berlin Heidelberg: Springer-Verlag; 2009: 667-692.

3. Yap HK, Han EJS, Heng CK, Cong WK. Risk factors for steroid dependency in children with idiopathic nephrotic syndrome. Pediatr Nephrol. 2001;16:1049-1052.

4. Wong W. Idiopathic nephrotic syndrome in New Zealand children, demographic, clinical features, initial management and outcome after twelve-month follow up: results of a three-year national surveillance study. J Pediatr Child Health. 2007;43:337-341. 
5. Kari JA, Halawani M, Mokhtar G, Jalalah SM, Anshasi W. Pattern of steroid resistant nephrotic syndrome in children living in the kingdom of Saudi Arabia: a single center study. Saudi J Kidney Dis Transpl. 2009; 20(5):854-857.

6. Hogg R, Middelton J, Vehaskari VM. Focal segmental glomerulosclerosisepidemiology aspects in children and adults. Pediatr Nephrol. 2007;22: 183-186.

7. Bircan Z, Yilmaz AY, Kater S, Vitrinel A, Yildirim M. Childhood idiopathic nephrotic syndrome in Turkey. Pediatr Int. 2002;44: 608-611.

8. McKinney PA, Feltbower RG, Brocklebank JT, Fitzpatrick MM. Time trends and ethnic patterns of childhood nephrotic syndrome in Yorkshire, UK. Pediatr Nephrol. 2001;16(12):1040-1044.

9. Chesney R. The changing face of childhood nephritic syndrome. Kidney Int. 2004;66:1294-1302.

10. Kari JA. Changing trends of histopathology in childhood nephrotic syndrome in western Saudia Arabia. Saudia Med J. 2002;23:317-321.

11. Gulati S, Sharma AP, Sharma RK, Gupta A. Changing trends of histopathology in childhood nephrotic syndrome. Am J Kidney Dis. 1999;34: 646-650.

12. Ahmadzadeh A, Derakhshan A, Hakimzadeh M, Zolfigol A. Idiopathic nephrotic syndrome in Iranian children. Indian Pediatr. 2008;45: $52-53$.

13. Safaei A, Maleknejad S. Spectrum of childhood nephrotic syndrome in Iran: a single center study. Indian J Nephrol. 2009;19(3):87-90.

14. Gulati S, Kher V, Sharma RK, Gupta A. Steroid response pattern in Indian children with nephrotic syndrome. Acta Pediatr. 1994;83(5): 530-533.

15. Bhimma R, Coovadia HM, Adhikarim. Nephrotic syndrome in South African children: changing perspectives over 20 years. Pediatr Nephrol. 1997;11(4):429-434.
16. Anochie I, Eke F, Okpere A. Childhood nephritic syndrome: change in pattern and response to steroids. J Nat Med Assoc. 2006;98(12): 1977-1981.

17. Mekahli D, Liutkus A, Ranchin B, et al. Long-term outcome of idiopathic steroid-resistant nephrotic syndrome: a multicenter study. Pediatr Nephrol. 2009;24:1525-1532.

18. Otukesh H, Otukesh S, Mojtahedzadeh M, et al. Management and outcome of steroid-resistant nephrotic syndrome in children. IJKD. 2009;3(4):210-216.

19. Chang JW, Tsai HL, Wang HH, Yang LY. Clinicopathological features and prognosis of Chinese children with idiopathic nephrotic syndrome between different age groups. Eur J Pediatr. 2009;168:1189-1194.

20. Sozeri B, Mir S, Mutlubas F, Sen S. The long-term results of pediatric patients with primary focal and segmental glomerulosclerosis. Saudi J Kidney Dis Transpl. 2010;21(1):87-92.

21. Kumar J, Gulati S, Sharma AP, Sharma RK, Gupta RK. Histopathological spectrum of childhood nephrotic syndrome in Indian children. Pediatr Nehrol. 2003;18:657-660.

22. Andenmatten F, Bianchetti MG, Gerber HA, Zimmermann A, Meregalli P, Oetliker OH. Outcome of idiopathic childhood nephrotic syndrome. A 20 year experience. Scand J Urol Nephrol. 1995; 29(1):15-29.

23. Gulati S, Sharma AP, Sharma RK, Gupta A, Gupta RK. Do current recommendations for kidney biopsy in nephrotic syndrome need modifications? Pediatr Nephrol. 2002;17:404-408.

24. Noer MS. Predictors of relapse in steroid- sensitive nephrotic syndrome. Southeast Asian J Trop Med Public Health. 2005;36(5):1313-1320.
Therapeutics and Clinical Risk Management

\section{Publish your work in this journal}

Therapeutics and Clinical Risk Management is an international, peerreviewed journal of clinical therapeutics and risk management, focusing on concise rapid reporting of clinical studies in all therapeutic areas, outcomes, safety, and programs for the effective, safe, and sustained use of medicines. This journal is indexed on PubMed Central, CAS

\section{Dovepress}

EMBase, Scopus and the Elsevier Bibliographic databases. The manuscript management system is completely online and includes a very quick and fair peer-review system, which is all easy to use. Visit http://www.dovepress.com/testimonials.php to read real quotes from published authors.

Submit your manuscript here: http://www.dovepress.com/therapeutics-and-clinical-risk-management-journal 